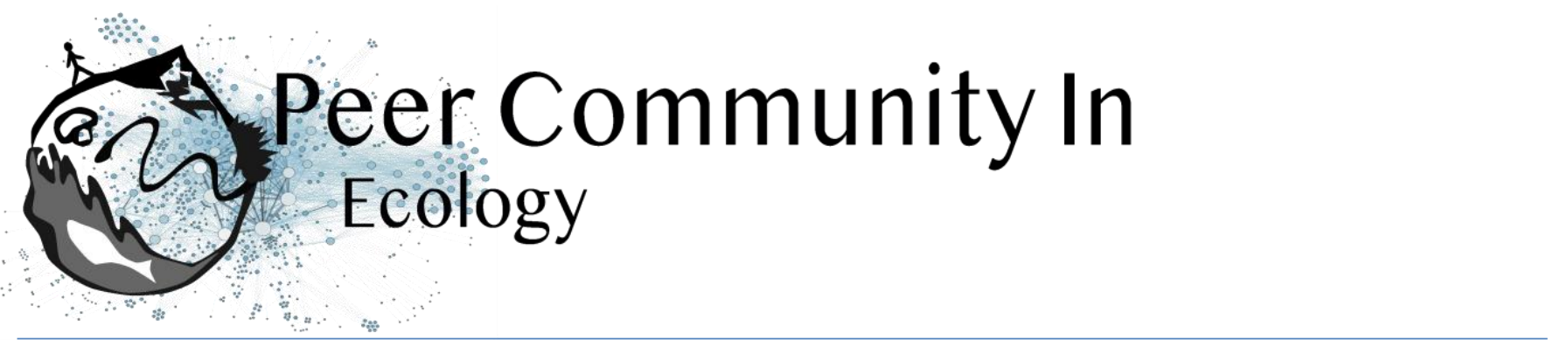

\title{
Dispersal: from "neutral" to a state- and context-dependent view
}

Emanuel A. Fronhofer based on reviews by 2 anonymous reviewers

\section{A recommendation of:}

Investigating sex differences in genetic relatedness in great-tailed grackles in Tempe, Arizona to infer potential sex biases in dispersal

Sevchik, A., Logan, C. J., McCune, K. B., Blackwell, A., Rowney, C. and Lukas, D(2021), EcoEvoRxiv, osf.io/t6beh, ver. 5 peer reviewed and recommended by PCI Ecology10.32942/osf.io/t6beh

\section{Open Access}

Published: 13 March 2021

Copyright: This work is licensed under the Creative Commons Attribution-NoDerivatives 4.0 International License. To view a copy of this license, visit http://creativecommons.org/licen ses/by-nd/4.0/
Submitted: 24 August 2020, Recommended: 10 March 2021

\section{Recommendation}

Traditionally, dispersal has often been seen as "random" or "neutral" as Lowe \& McPeek (2014) have put it. This simplistic view is likely due to dispersal being intrinsically difficult to measure empirically as well as "random" dispersal being a convenient simplifying assumption in theoretical work. Clobert et al. (2009), and many others, have highlighted how misleading this assumption is. Rather, dispersal seems to be usually a complex reaction norm, depending both on internal as well as external factors. One such internal factor is the sex of the dispersing individual. A recent review of the theoretical literature (Li \& Kokko 2019) shows that while ideas explaining sex-biased dispersal go back over 40 years this state-dependency of dispersal is far from comprehensively understood.

Sevchik et al. (2021) tackle this challenge empirically in a bird species, the greattailed grackle. In contrast to most bird species, where females disperse more than males, the authors report genetic evidence indicating male-biased dispersal. The authors argue that this difference can be explained by the great-tailed grackle's social and mating-system.

Dispersal is a central life-history trait (Bonte \& Dahirel 2017) with major consequences for ecological and evolutionary processes and patterns. Therefore, studies like Sevchik et al. (2021) are valuable contributions for advancing our understanding of spatial ecology and evolution. Importantly, Sevchik et al. also lead to way to a more open and reproducible science of ecology and evolution. The authors are among the pioneers of preregistering research in their field and their way of doing research should serve as a model for others.

\section{References}

Bonte, D. \& Dahirel, M. (2017) Dispersal: a central and independent trait in life history. Oikos 126: 472-479. doi: https://doi.org/10.1111/oik.03801

Clobert, J., Le Galliard, J. F., Cote, J., Meylan, S. \& Massot, M. (2009) Informed dispersal, heterogeneity in animal dispersal syndromes and the dynamics of 
spatially structured populations. Ecol. Lett.: 12, 197-209. doi: https://doi.org/10.1111/j.14610248.2008.01267.x

Li, X.-Y. \& Kokko, H. (2019) Sex-biased dispersal: a review of the theory. Biol. Rev. 94: 721-736. doi: https://doi.org/10.1111/brv.12475

Lowe, W. H. \& McPeek, M. A. (2014) Is dispersal neutral? Trends Ecol. Evol. 29: 444-450. doi: https://doi.org/10.1016/j.tree.2014.05.009

Sevchik, A., Logan, C. J., McCune, K. B., Blackwell, A., Rowney, C. \& Lukas, D. (2021) Investigating sex differences in genetic relatedness in great-tailed grackles in Tempe, Arizona to infer potential sex biases in dispersal. EcoEvoRxiv, osf.io/t6beh, ver. 5 peer-reviewed and recommended by Peer community in Ecology. doi: https://doi.org/10.32942/osf.io/t6beh

Cite this recommendation as:

Emanuel A. Fronhofer (2021) Dispersal: from "neutral" to a state- and context-dependent view. Peer Community in Ecology, 100074. 10.24072/pci.ecology.100074

\section{Reviews}

Toggle reviews

\section{Revision round \#3}

2021-03-02

\section{Author's Reply}

Download author's reply (PDF file)

Dear Dr. Fronhofer, reviewers, and the PCI Ecology Managing Board,

Thank you for providing this helpful feedback!

We made the changes listed below in the attached pdf to your and the reviewers' comments. The revised manuscript and associated files can be found at: https://ecoevorxiv.org/t6beh/

For an easier to read version of the final manuscript (including interactive code), please see: http://corinalogan.com/Preregistrations/gdispersal_manuscript.html.

As before, the version-tracked versions are in rmarkdown at GitHub: the preregistration (https://github.com/corinalogan/grackles/blob/master/Files/Preregistrations/gdispersal.Rmd) and the final

manuscript (https://github.com/corinalogan/grackles/blob/master/Files/Preregistrations/gdispersal_manuscript.R md). In case you want to see the history of track changes for these documents at GitHub, click the links and then click the "History" button on the right near the top. From there, you can scroll through our comments on what was changed for each save event and, if you want to see exactly what was changed, click on the text that describes the change and it will show you the text that was replaced (in red) next to the new text (in green).

Photo credit goes to Corina Logan (CC-BY 4.0).

Please let us know if you have any questions or need further information. Many thanks for your attention!

All our best, 


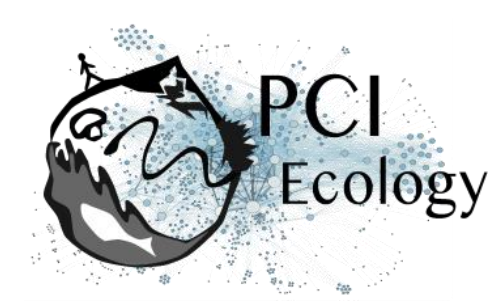

Gus, Corina, Kelsey, Aaron, Carol \& Dieter

\section{Decision round \#3}

Dear Mr. Sevchik, Dear Dr. Lukas,

thank you for your revisions. As you will see the referee is globally satisfied with the changes. Before I proceed to the recommendation of your manuscript, I would like you to go through one last round of revisions to address the referee's remaining concerns as well as some minor points listed below. I am looking forward to receiving a revised version of your preprint.

Sincerely yours,

Emanuel A. Fronhofer

Minor comments:

Line 161: "GiHhub" should be "GitHub"

Page 6: "Figure 1: Figure 1." should be "Figure 1:"

line 217: "loci" should be singular

line 240: "(Wang 2002)" should be "Wang (2002)". Same comment holds for the two reference in lines 241-242.

line 244-245: Year missing after "Queller \& Goodnight" and "Wang".

line 271: delete second bracket after "(Sutherland et al. (2000))". Same comment holds for a majority of reference in the paragraph lines 281-295.

\section{Preprint DOI: https://doi.org/10.32942/osf.io/t6beh}

\section{Reviewed by anonymous reviewer, 2021-02-18 09:44}

I have now read the third version of this manuscript which I reviewed twice in the past. The authors have taken in consideration all of our comments, and I find it particularly useful to have more natural history insight on the focal species. I am now happy to recommend this study.

I do have two remaining regrets, that are however not major and should not interfere with a recommendation of this manuscript. First, I still think that there can be some confusion about what is 'expected at random' (line 23) when evaluating whether average relatedness among individuals from a given site is high or not. Second, if the theoretical context outlined in the abstract is the resourcedefence based monogamous mating system it could be clearer how this context is linked to the prediction on sex-biased dispersal. In the introduction, it is explained that based on this hypothesis, the focal species might not display the usual female-biased dispersal found in birds (L64-65, L77-78) and yet 1 . The main hypothesis is that the focal species will display female-bias dispersal (one could expect the reverse based on the Introduction), and 2. The main result that males disperse further than females could be looped back more explicitly to the species mating system in the abstract (e.g. L31 replacing 'together with' by 'in line with'). 


\title{
Revision round \#2
}

\author{
2021-01-12
}

\section{Author's Reply}

Download author's reply (PDF file)

Dear Dr. Fronhofer, reviewers, and the PCI Ecology Managing Board,

Thank you for providing this helpful feedback! We made the changes listed in the attached pdf in response to your and the reviewers' comments. The revised manuscript and associated files can be found at: https://ecoevorxiv.org/t6beh/

We changed the structure according to your suggestion, and now provide a list with all changes from the preregistered protocol in the section "Deviations from the preregistration", and we include a description of all final analyses, explaining any changes, in the Methods section.

For an easier to read version of the final manuscript (including interactive code), please see: http://corinalogan.com/Preregistrations/gdispersalmanuscript.html. As before, the version-tracked versions are in rmarkdown at GitHub: the preregistration (https://github.com/corinalogan/grackles/blob/master/Files/Preregistrations/gdispersal.Rmd) and the final manuscript (https://github.com/corinalogan/grackles/blob/master/Files/Preregistrations/gdispersa/manuscript.Rm d). In case you want to see the history of track changes for these documents at GitHub, click the links and then click the "History" button on the right near the top. From there, you can scroll through our comments on what was changed for each save event and, if you want to see exactly what was changed, click on the text that describes the change and it will show you the text that was replaced (in red) next to the new text (in green). Photo credit goes to Corina Logan (CC-BY 4.0).

Please let us know if you have any questions or need further information. Many thanks for your attention!

All our best, Gus, Corina, Kelsey, Aaron, Carol \& Dieter

\section{Decision round \#2}

Dear Mr. Sevchik, Dear Dr. Lukas,

thank you for your revisions. As you will see both referees and I appreciate your efforts very much. Nevertheless, both referees have some major and some minor points that you should take into account when revising your manuscript.

Besides these points, I would like to draw you attention especially to one minor comment that may represent some additional work. One referee notes: "Will this preregistration mention appear in the final ms? Are details in this preregistration essential for the good understanding of this $\mathrm{ms}$ ? If so I feel that it would be better to have them included in the present ms rather than for the reader to have to download also the preregistration document. Reading the methods section, it feels like in fact it has been updated, hence I think it would be clearer to simply remove the preregistration mention, but I will let the Editor decide on this."

$\mathrm{PCl}$ does not (yet) have guidelines regrading how preprints that have preregistrations should be formatted. Nevertheless, one important aim, regardless of any guidelines, should be clarity and it seems that in its current format the manuscript could gain clarity by some restructuring. I would like to encourage you to make the manuscript read more like a self-sufficient entity and avoid the impression that the reader should check the preregistration. This being said, you should definitely refer to the preregistration and include the section on deviations as you are currently doing.

Minor points: The links included in the manuscript should all be DOls, otherwise they might not be stable and the manuscript may not be reproducible in some years time (e.g., line 102-103, code after 


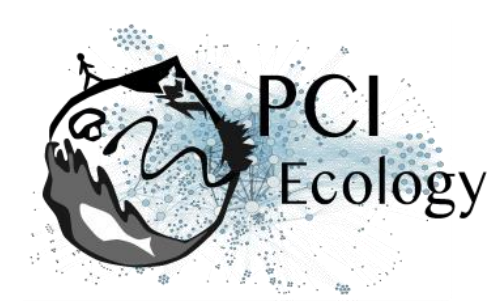

lines 277). You could use Zenodo, for instance, to generate DOls. Zenodo also allows for a nice integration with GitHub. Please check all of your links throughout the manuscript.

Line 168: symbol seems to be missing after "...many (R"

I suggest revising your preprint in light of the referees' comments, accompanied by a detailed response to their criticism. I am looking forward to receiving a revised version of your preprint.

Sincerely yours, Emanuel A. Fronhofer

Preprint DOI: https://doi.org/10.32942/osf.io/t6beh

Reviewed by anonymous reviewer, 2021-01-06 15:00

Download the review (PDF file)

\section{Reviewed by anonymous reviewer, 2020-12-14 20:31}

In this revised version of "Investigating sex differences in genetic relatedness in great-tailed grackles in Tempe, Arizona to infer potential sex biases in dispersal", the authors have addressed most of the comments made on a previous version of the ms; or adequately explained why they have not followed the reviewer or editor's suggestions (except for one very minor one, see below). The different text or figure editions and additions have helped clarifying some points. Only the part about relatedness comparison in different subsamples still needs some work, as I suggest below. Besides this point, I only have a few minor comments.

Abstract, I.29-30: «These relatedness results suggest that, unlike most other bird species, female great-tailed grackles appear to have hatched and remained at this site, while males disperse to new areas. " This sentence should be moderated a little, as the results do not show that all females stay and on-site, and all males disperse.

I.96: indivlduals

I.158: an extra "the" before " 5 individuals"

I.162: the estimated value of expected heterozygosity should be added

I.164: the authors forgot to use a mathematical expression instead of words in this sentence (editor comment \#5)

I.347 to 349: it is still unclear what was compared to what, in that paragraph. I suggest this rewording: "We compared (i) the observed average relatedness among the 37 females in our sample with the relatedness in the 10000 random samples of 37 individuals from both sexes; (ii) the observed average relatedness among the 15 males in our sample with the relatedness in the 10000 random samples of 15 individuals from both sexes; (iii) the observed average relatedness among the 15 males in our sample with the relatedness in the 10000 random samples of 15 females." Also to clarify these same analyses: I.173: add "from the whole sample" after "we randomly drew 37 individuals" And it would be helpful to the reader to add more explanations of why the authors conclude (as they do at the beginning of the discussion) that "the mean level of average genetic relatedness is lower among males compared to females", while their test comparing male and female relatedness suggests there is no difference (i.e. explain again the issue of lack of power in the samples made of 15 individuals in the interpretation part of the $\mathrm{ms}$ ).

I think Fig 2 and 3 are (in part) redundant, but l'll leave it up to the authors to decide whether to remove one or not. 


\title{
Revision round \#1
}

\author{
2020-10-06
}

\author{
Author's Reply \\ Download author's reply (PDF file) \\ Dear Emanuel A. Fronhofer,
}

We are grateful for the helpful comments on our manuscript "Investigating sex differences in genetic relatedness in great-tailed grackles in Tempe, Arizona to infer potential sex biases in dispersal". We have revised our manuscript accordingly. A pdf copy of the revised manuscript can be found at https://ecoevorxiv.org/t6beh. Alternatively for reading on screen we provide an html version at: http://corinalogan.com/Preregistrations/gdispersal_manuscript.html .

As before, the version-tracked version is in rmarkdown at GitHub: https://github.com/corinalogan/grackles/blob/master/Files/Preregistrations/gdispersal_manuscript.R md. In case you want to see the history of track changes for these documents at GitHub, click the links and then click the "History" button on the right near the top. From there, you can scroll through our comments on what was changed for each save event and, if you want to see exactly what was changed, click on the text that describes the change and it will show you the text that was replaced (in red) next to the new text (in green).

Our detailed replies to your and the reviewers' comments are in the attached pdf. Please let us know if you need anything else.

Photo credit goes to Corina Logan (CC-BY 4.0).

We look forward to hearing back from you!

With best wishes, Dieter Lukas - on behalf of all authors.

\section{Decision round \#1}

Dear Mr. Sevchik, Dear Dr. Lukas,

thank you very much for submitting your preprint "Investigating sex differences in genetic relatedness in great-tailed grackles in Tempe, Arizona to infer potential sex biases in dispersal" to $\mathrm{PCl}$ Ecology. It is great to see preregistrations leading to preprints.

Two referees have reviewed your work and I agree very much with their points which you will find below. I would like to highlight that both referees have issues with some of the presentation, for example, the end of the abstract. I agree very much and would suggest that you reformulate as suggested. This holds for the entire text.

Both referees feel that there is some need for more biological and behavioural context and some methodological questions are also raised.

The first referee also has some points related to visualization and I find myself in agreement. I suggest replacing Fig. 1 with a 2-panel figure that shows males and females, for example. The figure caption should also include a quantitative and not qualitative statement ("small number of random draws"). Along similar lines of thought, I was wondering whether Fig. 2 could be adapted to actually show what is described in the paragraph above. Finally, for readers who are less familiar with the methods you use, I would suggest explaining why relatedness can be negative.

Minor points: line 15: "were hatched" should be "hatched" lines 136-143: Please correct brackets for references (See also lines 302-305.). line 164: Please use a mathematical expression for this.

figures: "Figure X" is always repeated twice. Please correct this. 
I suggest revising your preprint in light of the referees' comments, accompanied by a detailed response to their criticism. I am looking forward to receiving a revised version of your preprint.

Sincerely yours, Emanuel A. Fronhofer

Preprint DOI: https://doi.org/10.32942/osf.io/t6beh

\section{Reviewed by anonymous reviewer, 2020-09-23 08:12}

Download the review (PDF file)

\section{Reviewed by anonymous reviewer, 2020-09-18 17:20}

In this study, Sevchik and collaborators use genetics to investigate sex-biased dispersal at a local spatial scale in great-tailed grackles. Based on ddRadSeq genotypes, they use three sets of analyses to compare among sexes their degree of relatedness, the geographic distances of related individuals, and the patterns of spatial autocorrelation. These analyses allow them to show that contrary to most bird species, dispersal is male-biased in great-tailed grackles. The sample size is limited but the genetic data are substantial. The methods used are faithful to the description made in the pre-registration, which was recommended. Overall the ms is nicely written, and I appreciated that hypotheses and clear predictions were presented. My comments mostly concern sentences or paragraphs which clarity can be improved, as detailed below.

Abstract, last sentence, «Our findings show that reduced resource competition might facilitate female philopatry and that prior knowledge of an area does not appear to be a prerequisite for male great-tailed grackles to establish breeding territories": The beginning of the sentence needs to be reformulated, as this study was not designed to assess the effect of resource competition on dispersal.

Main hypothesis: "Based on the argument that males are expected to be philopatric when they defend resources beneficial to females": In the paragraph describing the species behavior it is suggested that only some males defend territories. So it is surprising for the reader that the formulation of the main hypothesis relies on the idea that males defend resources. It is only when reading the alternative hypotheses that the reader understands that the different hypotheses are based on different aspects of the species behaviour. I suggest slightly reformulating the main hypothesis to make clear that it really means "given that some males defend resources, in which case males are expected to be philopatric and females to disperse to avoid mating with relatives, $(\ldots)$.

Alternative hypothesis 2: "The polygamous mating system of great-tailed grackles, where females might be able to choose among potential males, might reduce a female's risk of mating with their father or brother." This argument is valid only if females can discriminate relatives from non relatives. Has this been demonstrated in this species?

I.108: IN the methods below.

I.119-124: It is currently difficult to distinguish the parameters from Thrasher et al. 2018 and those specific to the study presented in this $\mathrm{ms}$, because of missing brackets and long sentences with semi-columns. Some rewording would help.

Results, I.159-164: It would be informative to add an estimate of the total size of the population, if it is know. That would allow to assess how representative the genetic sample is.

Results, I.161-164: How was the probability of identity of siblings estimated? (And heterozygosity compared to expected in a population with random mating? - Do the authors simply mean HW equilibrium, here (but they would have forgotten other conditions of HW equilibrium))? This piece of information is missing from the method section. 


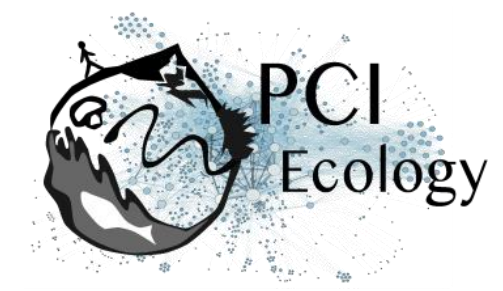

Analysis i: I.318-326: "We performed 10,000 random draws of 15 individuals either from among the females or from among all individuals and of 37 individuals from among all individuals, and generated distributions of average relatedness among these samples. We assessed whether the observed average relatedness among the 15 males or the 37 females in our sample is higher than what is observed in the majority of random samples." This description is a bit confusing, as it is difficult to understand what was compared to what. Besides, in the results section, it seems that in the end only one random distribution was used (10,000 random draws of 37 individuals from among all individuals). I think comparing the observed relatedness to this random distribution is indeed enough, and that the authors should simplify the method description by removing mentions to the other random distributions.

Fig.1: I suggest adding arrows showing also the average relatedness of males (and of the whole sample).

I.181: Did the authors mean 8 female dyads, instead of 7 ?

I.186: "larger" missing after "were found at".

Fig.2: Also add a dotted line to show the 0.125 relatedness level.

Results of the spatial autocorrelation analysis: They would be much easier to read if they were presented as a figure (spatial autocorrelation coefficient as a function of geographic distance, with standard errors allowing to assess the significance of the autocorrelation coefficients) instead of a table.

Dependent variable, I.307: average relatedness is not the only dependent variable (e.g. analysis ii, geographic distances among relatives).

Between I.312 and 313, title of the 3rd section of analyses: females should be replaced with males.

Between I.327 and 328, 3rd title: remove "and the juvenile individuals"; 4th title: eXCLude

I.329-336: The explanation of the generation of random distributions of geographic distances is confusing. It is clearer in I.146-151. Maybe it would be better to first explain the process for one sex, and then the other. Indeed, what is making the paragraph confusing is that both procedures are mixed in the same sentence, which makes it difficult to understand what was compared to what in the end. 